See referenced original article on pages 1101-12, this issue.

The author is a member of the Speaker's Bureau and has received an unrestricted educational grant from Schering Oncology Biotech.

Address for reprints: Vernon K. Sondak, M.D., Professor of Surgery, Division of Surgical Oncology, University of Michigan Comprehensive Cancer Center, Ann Arbor, MI 48109-0932; Fax: (734) 647-9647; E-mail: vsondak@umich.edu

Received May 28, 2002; accepted May 28, 2002.

\section{How Does Interferon Work? Does it Even Matter?}

\author{
Vernon K. Sondak, M.D. \\ Division of Surgical Oncology, University of Michigan Comprehensive Cancer Center, Ann Arbor, \\ Michigan.
}

A djuvant therapy for patients with melanoma using interferon $\alpha$ remains controversial despite numerous randomized clinical trials. ${ }^{1,2}$ There can be no doubt that interferon $\alpha$ works-that is, it has biologic activity sufficient to alter the natural history of resected melanoma. Multiple randomized trials have demonstrated that adjuvant interferon prolongs recurrence free survival compared with observation $^{3-7}$ or compared with a defined antigen vaccine. ${ }^{8,9}$ The effect on overall survival is more contentious-only two studies have demonstrated a significant effect on overall survival, ${ }^{3,8}$ and only one of those studies included an untreated control group. ${ }^{3}$ Nonetheless, prolonged recurrence free survival is clear-cut evidence of a biologic effect of the therapy. From a clinical standpoint, however, this demonstrated recurrence free survival and presumptive overall survival benefit must be weighed against the substantial toxicity of therapy. It is interesting to note that, in the ongoing debate over the clinical role of adjuvant interferon $\alpha$, little attention has been paid to the question of how interferon works.

Most clinicians seemingly accept at face value the notion that interferon $\alpha$ acts as an immunomodulator, augmenting the host immune response to melanoma-associated antigens on residual tumor cells. However, because interferon $\alpha$ has multiple known activities, ${ }^{10}$ this simple notion may not be as straightforward as it first appears. Perhaps the most salient reason to question a purely immunomodulatory role for interferon $\alpha$ is the failure of interferon $\gamma$ to prolong recurrence free survival in two randomized trials. ${ }^{11,12}$ Interferon $\gamma$ at the doses administered in those two trials is a documented immunomodulator, ${ }^{13,14}$ one that many tumor immunologists would say is superior to interferon $\alpha$. Thus, if the mechanism of action were purely immunostimulatory, then we would anticipate an equal or greater effect for interferon $\gamma$. However, even if we accept the fact that the mechanism of action of interferon $\alpha$ is uncertain, why should we care?

A better understanding of the mechanism of action of adjuvant interferon $\alpha$ may help with three unresolved but closely interrelated clinical questions: 1) What is the optimum dose of interferon $\alpha$ ? 2) What is the optimum duration of therapy, and should therapy be stopped or continued in the event of a resectable recurrence? 3) How can we minimize the toxicity of interferon $\alpha$ without losing efficacy?

In this issue of Cancer, Kirkwood et al. ${ }^{15}$ present the results of a biologic companion trial to Intergroup Trial E1690, which compared high-dose and low-dose interferon $\alpha 2 \mathrm{~b}$ with observation after sur- 
gery. ${ }^{4}$ In some ways, the parent E1690 trial epitomizes the controversies that surround adjuvant interferon $\alpha$ : This trial confirmed the recurrence free survival impact of high-dose interferon seen in the original E1684 trial but failed to show any overall survival benefit. Low-dose interferon resulted in a transient but not statistically significant prolongation of recurrence free survival, but this effect diminished within 1-2 years of the end of therapy, and the recurrence free survival curve gradually came down to meet the curve of the observation arm. This "banana-shaped curve" has been seen with other low-dose interferon trials as well. ${ }^{16}$

A better understanding of the mechanism of action of interferon clearly would help us select the ideal dose. At one extreme, it has been suggested that interferon acts as an antiangiogenesis agent, and at least some preclinical data suggest that interferon $\alpha$ inhibits angiogenesis best at very low doses. ${ }^{17}$ Only one clinical trial has looked at very-low-dose interferon $\alpha$, in the range of putative optimal angiogenesis inhibition, and that trial showed no discernable impact on recurrence free survival. ${ }^{12}$ At the other extreme, it has been argued that the initial 1-month-long, daily, intravenous portion of the high-dose interferon regimen may be necessary and even sufficient. Two clinical trials currently underway are evaluating the 1-month intravenous regimen alone as adjuvant therapy (E1697 and the Sunbelt Melanoma Trial protocol $\mathrm{B}^{18}$ ). Neither trial, however, includes a comparison group receiving the entire 1-year intravenous and subcutaneous regimen. Low-dose interferon for up to 2 years, as tested in E1690, has worked either transiently ${ }^{4,16,19}$ or not at $\mathrm{all}^{20}$ and seems to be a dead end, at least for high-risk patients. One recently concluded trial of great interest is a European Organization for Research and Treatment of Cancer (EORTC) trial of intermediate/highdose interferon $\alpha$, with no intravenous component, that reportedly is showing early evidence of a significant prolongation of distant disease free survival for patients who are treated with $10 \mathrm{MU}$ subcutaneously daily for 5 days per week for 4 weeks followed by $5 \mathrm{MU}$ subcutaneously three times each week for 2 years versus observation. ${ }^{21}$

Understanding the mechanism of action also may help us decide how long interferon therapy should be used. The concept of decreasing the duration of therapy to 1 month, relying exclusively on the intravenous induction phase of the current year-long regimen, was discussed previously. Another strategy is to increase the duration of therapy while lowering the dose-like the above-cited EORTC trial of 2 years of intermediate/high-dose therapy. Yet another approach, also in a clinical trial by the EORTC, is truly long-term therapy
(5 years of treatment) using longer acting, pegylated interferon. It is noteworthy that toxicity with either the 1-month intravenous regimen or the $2-5$-year intermediate dose to intermediate/high-dose regimens seems to be less than the toxicity seen with the 1-year, high-dose regimen.

Finally, clinicians frequently face the scenario of a patient who experiences a disease recurrence while on interferon, but the recurrence is localized and resectable (frequently at the primary site and/or the regional lymph node basin). In these instances, the physician and patient must decide whether or not to continue the interferon. In the United States, many (but not all) physicians routinely recommend discontinuing interferon after any recurrence, even if it is localized. In European clinical trials, the practice generally has been to continue interferon until the patient develops a disseminated, distant recurrence. If interferon $\alpha$ functions as an antimetastatic agent (either by angiogenesis inhibition or by other, as yet undefined mechanisms), then it would seem unwise to discontinue therapy for a local or regional recurrence. If interferon $\alpha$ functions by purely immunologic mechanisms, then the implications are less clear, and arguments could be made both ways.

Faced with these questions, how do the observations of Kirkwood and his colleagues help us? First, it must be recognized that these are important initial observations, not final answers. The authors studied only immunologic end points and did not establish any parameters that clearly were linked with a favorable outcome from therapy. The authors properly point out that the numbers of patients (representing only $21 \%$ of the entire study population) were too small to exclude potentially relevant associations between interferon effects and outcome. Conversely, the authors did identify several parameters that were impacted differentially by high-dose versus low-dose interferon, most notably, T-cell and natural killer cell function, T-cell subset distribution, major histocompatibility complex class II expression, and adhesion molecule expression. These observations set the stage for future, hypothesis-based explorations of interferon $\alpha$ function that can begin to answer relevant clinical questions and guide future clinical trial design.

One final-and highly speculative-note: Perhaps the most provocative (but very preliminary) finding in this study was the strong trend $(P=0.06)$ for a negative interaction between human leukocyte antigen A2 (HLA-A2) status and the effect of interferon. This relation should be evaluated further in other interferon trials. If it is substantiated, then it would certainly require a rethinking of mechanisms of action. Conversely, it could allow targeting of therapy to those 
patient populations most likely to benefit and the development of other adjuvant therapies (such as vaccines) for the rest. Even if this preliminary observation about HLA-A2 status ultimately is proven incorrect, it should serve as a highly visible reminder of the potential value of a better understanding of how (and for whom) interferon works. For clinicians-and clinical trialists-that indeed does matter.

\section{REFERENCES}

1. Dawes M, Lens MB. Interferon alfa therapy for malignant melanoma: a systemic review of randomized controlled trials. J Clin Oncol. 2002;20:1818-1825.

2. Sabel MS, Sondak VK. Pros and cons of adjuvant interferon in the treatment of melanoma. Oncol Spectrums. 2002;3: $264-270$.

3. Kirkwood JM, Strawderman MH, Ernstoff MS, et al. Interferon alfa-2b adjuvant therapy of high-risk resected cutaneous melanoma: the Eastern Cooperative Oncology Group trial EST 1684. J Clin Oncol. 1996;14:7-17.

4. Kirkwood JM, Ibrahim JG, Sondak VK, et al. High- and low-dose interferon alfa-2b in high-risk melanoma: first analysis of Intergroup trial E1690/S9111/C9190. J Clin Oncol. 2000;18:2444-2454.

5. Rusciani L, Petraglia S, Alotto M, Calvieri S, Vezzoni G. Postsurgical adjuvant therapy of melanoma: evaluation of a 3 -year randomized trial with recombinant interferon- $\alpha$ after 3 and 5 years of follow-up. Cancer. 1997;79:2354-2360.

6. Grob JJ, Dreno B, de la Salmoniere P, et al. Randomised trial of interferon alpha-2a as adjuvant therapy in resected primary melanoma thicker than $1.5 \mathrm{~mm}$ without clinically detectable node metastases. French Cooperative Group on Melanoma. Lancet. 1998;351:1905-1910.

7. Pehamberger H, Soyer HP, Steiner A, et al. Adjuvant interferon alfa-2a treatment in resected primary Stage II cutaneous melanoma. Austrian Malignant Melanoma Cooperative Group. J Clin Oncol. 1998;16:1425-1429.

8. Kirkwood JM, Ibrahim JG, Sosman JA, et al. High-dose interferon alfa-2b significantly prolongs relapse-free and overall survival compared with the GM2-KLH/QS-21 vaccine in patients with resected Stage IIB-III melanoma: results of Intergroup Trial E1694/S9512/C509801. J Clin Oncol. 2001; 19:2370-2380.

9. Kirkwood JM, Ibrahim J, Lawson DH, et al. High-dose interferon alfa-2b does not diminish antibody response to GM2 vaccination in patients with resected melanoma: results of the multicenter Eastern Cooperative Oncology Group Phase II trial E2696. J Clin Oncol. 2001;19:1430-1436.

10. Pfeffer LM, Dinarello CA, Herberman RB, et al. Biological properties of recombinant alpha-interferons: 40th anniversary of the discovery of interferons. Cancer Res. 1998;58: 2489-2499.

11. Meyskens FL, Kopecky K, Taylor CW, et al. Randomized trial of adjuvant human interferon gamma versus observation in high-risk cutaneous melanoma. J Natl Cancer Inst. 1995;87: 1710-1713.

12. Kleeborg U, Broecker EB, Chartier C, et al. EORTC 18871 adjuvant trial in high risk melanoma patients: IFN $\alpha$ vs IFN $\gamma$ vs Iscador vs observation [abstract]. Eur J Cancer. 1999; 35(Suppl 4):264.

13. Jaffe HS, Herberman RB. Rationale for recombinant human interferon-gamma adjuvant immunotherapy for cancer. J Natl Cancer Inst. 1988;80:616-618.

14. Maluish AE, Urba WJ, Longo DL, et al. The determination of an immunologically active dose of interferon-gamma in patients with melanoma. J Clin Oncol. 1988;6:434-445.

15. Kirkwood JM, Richards T, Zarour HM, et al. Immunomodulatory effects of high-and low-dose IFNa2b in patients with high-risk resected melanoma: the E2690 laboratory corollary of Intergroup adjuvant trial E1690. Cancer. 2002;95:11011112.

16. Cascinelli N, Bufalino R, Morabito A, et al. Results of adjuvant interferon study in WHO melanoma programme. Lancet. 1994;343:913-914.

17. Fidler IJ. Angiogenesis and cancer metastasis. Cancer J Sci Am. 2000;6(Suppl 2):S134-S141.

18. McMasters KM. The Sunbelt Melanoma Trial. Ann Surg Oncol. 2001;8(Suppl 9):41-43.

19. Cameron DA, Cornbleet MC, Mackie RM, et al. Adjuvant interferon alpha $2 \mathrm{~b}$ in high risk melanoma-the Scottish study. Br J Cancer. 2001;84:1146-1149.

20. Hancock BW, Wheatley K, Harrison G, Gore M. Aim highadjuvant interferon in melanoma (high risk), a United Kingdom Co-ordinating Committee on Cancer Research (UKCCCR) randomised study of observation versus adjuvant low dose extended duration interferon alpha-2a in high risk resected malignant melanoma [abstract]. Proc Am Soc Clin Oncol. 2001;20:349a.

21. Eggermont AMM, Kleeberg U, Ruiter DJ. European Organization for Research and Treatment of Cancer Melanoma Group trial experience with more than 2,000 patients, evaluating adjuvant treatment with low or intermediate doses of interferon alpha-2b. In: Perry MC, editor. American Society of Clinical Oncology 2001 educational book. Baltimore: Lippincott, Williams, Wilkins, 2001:88-93. 